\title{
VIDEOS PARA MEJORAR LAS HABILIDADES PRÁCTICAS Y CONOCIMIENTOS DE CUIDADORES DE PACIENTES CON ACCIDENTE CEREBROVASCULAR
}

\author{
Claudia María Sánchez-Huamash(1,a, César Cárcamo-Cavagnaro(1,b \\ 1 Facultad de Salud Pública y Administración, Universidad Peruana Cayetano Heredia, Lima, Perú. \\ a Licenciada Tecnóloga Médico en Terapia Física y Rehabilitación, magíster en Informática Biomédica en Salud Global; \\ b médico cirujano, doctor en Epidemiología.
}

El presente estudio forma parte de la tesis de Sánchez-Huamash C. Eficacia del uso de videos educativos para cuidadores de pacientes en fase subaguda del accidente cerebrovascular [tesis de Maestría]. Lima: Facultad Salud Pública y Administración, Universidad Peruana Cayetano Heredia; 2019.

\section{RESUMEN}

Objetivo: Evaluar el efecto de videos educativos para mejorar las habilidades prácticas y conocimientos de los cuidadores informales de pacientes con accidente cerebrovascular. Materiales y métodos: Estudio pretest/postest. Los participantes fueron diez cuidadores de 18 a 65 años y de ambos sexos de pacientes en fase subaguda del accidente cerebrovascular. Se desarrollaron ocho videos educativos relacionados a posicionamientos, movilizaciones y transferencias, que se implementaron en la casa de los cuidadores y/o pacientes a lo largo de tres días (en el primer día, tres videos; segundo día, tres videos; y tercer día, dos videos). Cada video fue visualizado en una tableta por 30 minutos. Las habilidades prácticas y conocimientos se evaluaron antes y después de cada video, y finalmente, la satisfacción de los cuidadores. Se usaron tres instrumentos, una lista de chequeo para evaluar las habilidades prácticas, un cuestionario para evaluar los conocimientos y otro para la satisfacción. Resultados: El puntaje de las habilidades prácticas incrementó de 21,6 a 56,1 puntos ( $\mathrm{p}<0,001)$ y los conocimientos de 11,6 a 21,6 puntos $(\mathrm{p}<0,001)$. Además, 7/10 de los cuidadores estaban muy satisfechos con los videos, 7/10 consideró fácil las palabras, 7/10 consideró fácil poner en práctica las indicaciones, 9/10 definitivamente recomendaría los videos y todos los consideraban útiles. Conclusión: Los videos educativos mejoran las habilidades prácticas y conocimientos de los cuidadores informales de pacientes con accidente cerebrovascular. Los videos educativos desarrollados podrían usarse para capacitar a los cuidadores sobre el manejo de estos pacientes.

Palabras clave: Accidente Cerebrovascular; Atención Subaguda; Servicios de Atención de Salud a Domicilio; Cuidadores; Fisioterapia; Película y Video Educativos; Aptitud; Satisfacción del Paciente (fuente: DeCS BIREME).

\section{VIDEOS TO IMPROVE THE SKILLS AND KNOWLEDGE OF STROKE PATIENTS' CAREGIVERS}

Citar como: Sánchez-Huamash CM, Cárcamo-Cavagnaro C. Videos para mejorar las habilidades prácticas y conocimientos de cuidadores de pacientes con accidente cerebrovascular. Rev Peru Med Exp Salud Publica. 2021;38(1):41-8. doi: https://doi. org/10.17843/rpmesp.2021.381.6130.

Correspondencia: Claudia María Sánchez Huamash; Av. Honorio Delgado 430, San Martin de Porres, Lima 15102, Perú; claudia.sanchez.h@upch.pe

Recibido: $04 / 07 / 2020$

Aprobado: 03/02/2021

En línea: 19/02/202

\section{ABSTRACT}

Objective: To evaluate the effect of educational videos to improve the practical skills and knowledge of stroke patients' informal caregivers. Materials and methods: Pretest/posttest study. The participants were 10 caregivers aged 18 to 65 years and of both sexes of patients in the subacute phase of stroke. We developed eight educational videos related to positioning, mobilizations and transfers, which were implemented in the caregivers' and/or patients' homes along three days (on the first day, three videos; second day, three videos; and third day, two videos). Each video was viewed on a tablet for 30 minutes. We evaluated skills and knowledge before and after each video, and finally, the caregivers' satisfaction. We used three instruments, a checklist to assess practical skills, a questionnaire to assess knowledge, and another for satisfaction. Results: The practical skills score increased from 21,6 to 56,1 points $(\mathrm{p}<0,001)$ and knowledge from 11,6 to 21,6 points ( $\mathrm{p}<0,001$ ). $7 / 10$ of the caregivers were very satisfied with the videos, 7/10 considered the words easy, 7/10 considered easy to put the indications into practice, $9 / 10$ would definitely recommend the videos, and everyone considered them useful. Conclusion: Educational videos improve the practical skills and knowledge of stroke informal caregivers. Developed educational videos could be successful in training caregivers on the management of these patients.

Keywords: Stroke; Subacute Care; Home Care Services; Caregivers; Physical Therapy Specialty; Instructional Film and Video; Aptitude, Knowledge; Patient Satisfaction (source: MeSH NLM). 


\section{INTRODUCCIÓN}

Los pacientes que sufrieron un accidente cerebrovascular (ACV) y sobrevivieron tienen secuelas que en más del 80\% son motoras, dentro de ellas la hemiplejia ${ }^{(1)}$. Por lo tanto, serán pacientes dependientes y requerirán un cuidador, quien a menudo es un miembro de la familia que adapta sus propias responsabilidades y asume ese papel incluso sin tener la capacitación adecuada (cuidador informal). Los cuidadores juegan un papel muy importante, especialmente después del alta hospitalaria ${ }^{(2,3)}$, que por lo general coincide con la fase subaguda y es la etapa en que los pacientes logran la mayor parte de su recuperación ${ }^{(4)}$. La educación del cuidador es crucial para reducir los riesgos para su propia salud física y mental ${ }^{(5)}$ y para prevenir complicaciones y promover la recuperación del paciente ${ }^{(6)}$.

Para la mayoría de los cuidadores y pacientes, el alta hospitalaria es uno de los momentos más difíciles. El cuidador no se siente preparado para regresar a casa, principalmente debido a la falta de conocimiento para lidiar con las secuelas. Además, han informado de la necesidad de recibir capacitación en tareas de atención específicas, como posicionamiento, movilizaciones y transferencias ${ }^{(2,7)}$. El posicionamiento, las movilizaciones y transferencias correctas protegen las articulaciones, proporcionan comodidad y previenen complicaciones como problemas respiratorios, úlceras de decúbito, dolor, contracturas, acortamientos e inflamación. Asimismo, promueven la recuperación y maximizan la función del paciente a través de la modulación del tono, información sensorial adecuada, mayor percepción espacial, estabilización de segmentos corporales y activación neuromuscular ${ }^{(8,9)}$.

En América Latina, la mayoría de los pacientes tienen varias limitaciones para acceder a los servicios de salud ${ }^{(10)}$. En el Perú, sobre todo en las zonas rurales, los sistemas de salud están saturados, tienen cobertura limitada y el acceso es retrasado, el número de especialistas es limitado y existe inequidad social ${ }^{(11)}$. Un estudio informó que en Lima ninguno de los cuidadores de pacientes que habían sufrido un accidente cerebrovascular había recibido orientación sobre el cuidado de sus pacientes después del alta hospitalaria ${ }^{(7)}$. Esta situación se agrava en zonas rurales, donde es menor el porcentaje que conoce y usa los servicios de rehabilitación, y es mayor el problema de accesibilidad relacionado al transporte, distancia y barreras físicas ${ }^{(12)}$.

El desarrollo de la tecnología representa nuevas oportunidades para mejorar la prestación de asistencia sanitaria. Es así que se han desarrollado intervenciones de $m$ Health o salud móvil como telemedicina, videoconferencias, envío de mensajes de texto, aplicaciones móviles, entre otros ${ }^{(12)}$. Además, se ha demostrado que los videos son herramientas educativas efectivas. Los videos dirigidos a pacientes con enfermedad pulmonar obstructiva crónica (EPOC) han mejorado el conocimiento y la técnica en el uso de inhaladores ${ }^{(13)}$. De

\section{MENSAJES CLAVE}

\begin{abstract}
Motivación para realizar el estudio: La capacitación de los cuidadores de pacientes con accidente cerebrovascular es crucial para la salud del mismo cuidador y la recuperación del paciente. Sin embargo, hay limitaciones para acceder a los servicios de salud, y sobre todo para este tipo de intervenciones, por lo que se requiere desarrollar y evaluar el efecto de estrategias y herramientas como videos educativos para dicha capacitación.
\end{abstract}

Principales hallazgos: Se desarrollaron videos educativos que mejoran las habilidades prácticas y conocimientos de los cuidadores de pacientes con accidente cerebrovascular.

Implicancias: Los videos pueden ser usados en la capacitación de estos cuidadores.

igual forma, los videos han aumentado los conocimientos de pacientes con $\mathrm{ACV}{ }^{(14)}$, cuidadores de pacientes con asma ${ }^{(15)}$, niños con epilepsia ${ }^{(16)}$ y pacientes en unidades de cuidados intensivos ${ }^{(17)}$. Por otro lado, el entrenamiento presencial entre los mismos cuidadores o con actores que hacen de pacientes, sumado a retroalimentación por parte del profesional de salud, han incrementado el conocimiento y autocompetencia de cuidadores de pacientes con demencia ${ }^{(18)}$, y la autoeficacia y preparación para el cuidado de los cuidadores de pacientes con cáncer ${ }^{(19)}$, de igual manera, los conocimientos y prácticas reportadas en cuidadores de pacientes con $\mathrm{ACV}^{(20)}$.

A pesar de que hay evidencia que demuestra la eficacia de la educación en cuidadores, tanto de forma presencial como con videos educativos, la mayoría de estudios evalúan los conocimientos, la autoeficacia o autocompetencia, no las técnicas o habilidades prácticas, sobre todo relacionadas al posicionamiento, movilizaciones o transferencias para pacientes que hayan sufrido un ACV. Además, es necesario desarrollar y evaluar intervenciones culturalmente enfocadas que puedan servir como nuevas formas de prestación de servicios de salud. El objetivo del estudio fue evaluar el efecto de los videos educativos para mejorar las habilidades prácticas y conocimientos de los cuidadores informales de pacientes con $\mathrm{ACV}$.

\section{MATERIALES Y MÉTODOS}

\section{Diseño y población de estudio}

Se realizó un estudio pretest/postest. Se reclutaron los participantes en el departamento de hospitalización y emergencias del Hospital Cayetano Heredia, Lima, Perú. Los criterios de inclusión fueron que sean cuidadores informales (familiares) de pacientes que hayan sufrido un $\mathrm{ACV}$ isquémico, que se encuentren en fase subaguda (desde los primeros 
siete días del inicio de los síntomas hasta los primeros tres meses) ${ }^{(4)}$, estables y tengan hemiplejia. Asimismo, que la edad de los cuidadores sea mayor a 18 y menor a 65 años. Se excluyeron a los cuidadores que tenían problemas cognitivos, auditivos o visuales graves o si sus pacientes no colaboraban debido a sus condiciones cognitivas, neurológicas o psiquiátricas; y/o tenían comorbilidades que afectaban la movilidad, como otros trastornos neurológicos, ortopédicos o traumatológicos.

\section{Videos educativos}

Los videos educativos presentaron actividades relacionadas al posicionamiento, movilizaciones y transferencias para un buen manejo de pacientes en fase subaguda del ACV. Los guiones de los videos se desarrollaron en base a literatura especializada ${ }^{(8,921,22)}$ y al asesoramiento de siete expertos: cuatro tecnólogos médicos en terapia física y rehabilitación, un geriatra, un neurólogo y un comunicador. Se grabaron los guiones y posteriormente los videos, cuyo elenco consistió en dos personajes: el investigador del estudio que actuaba como cuidador y un actor profesional como paciente. Tres tecnólogos médicos en terapia física y rehabilitación evaluaron los videos y dieron comentarios para mejorarlos. Finalmente, se obtuvo ocho videos educativos que duraban entre 1 minuto 15 segundos y 2 minutos 44 segundos, así como un video introductorio de 1 minuto 15 segundos para explicar principalmente qué lado se consideró afectado (video 0).

Los ocho videos corresponden a las siguientes actividades: video 1 - alineación del paciente en la posición echado boca arriba; video 2 - alineación en la posición echado de costado sobre el lado afectado; video 3 - alineación echado de costado sobre el lado sano; video 4 - cómo elevar las caderas del paciente cuando está echado boca arriba; video 5 - cómo girar al paciente sobre su lado afectado y sano; video 6 - cómo sentar al paciente en el borde de la cama desde la posición echada; video 7 - cómo trasladar al paciente de una cama a una silla; video 8-alineación del paciente cuando está sentado en una silla. Estos videos están disponibles en: https://www.youtube.com/playlist?list=PLXIX1w9QhG15NPlTb0TqSkeYvbp3NzFT_

\section{Instrumentos}

Para las habilidades prácticas (Material suplementario 1), se usó una lista de verificación de 68 ítems que fue administrada por el investigador. Para los conocimientos (Material suplementario 2), un cuestionario con 27 preguntas cerradas de selección simple, que fue completado por el cuidador. En ambos instrumentos, se asignó un punto para cada ítem/ respuesta correcta y cero para cada ítem/respuesta incorrecta. Para evaluar la satisfacción (Material suplementario 3), se usó un cuestionario con 7 preguntas cerradas de selección simple y 3 preguntas abiertas, que fue completado por el cuidador. Los instrumentos fueron desarrollados a partir de la literatura especializada usada para la elaboración de los videos. Posteriormente, los instrumentos fueron evaluados por cinco tecnólogos médicos en terapia física y rehabilitación, de acuerdo a los criterios de suficiencia, relevancia y claridad, luego se realizaron las correcciones respectivas. Finalmente, los instrumentos para evaluar los conocimientos y satisfacción se aplicaron a cinco posibles cuidadores con el objetivo de mejorar la semántica de las preguntas y alternativas, se hicieron las correcciones y se obtuvieron los instrumentos finales.

\section{Procedimientos}

En los servicios de hospitalización y emergencia del Hospital Cayetano Heredia se identificaron los casos de pacientes con $\mathrm{ACV}$ isquémico, se evaluaron si cumplían los criterios de selección, y en caso fuera así, se conversó personalmente o vía telefónica con un familiar para conocer quién sería el cuidador del paciente y a la vez verificar si cumplía los criterios de selección. Si el cuidador cumplía dichos criterios, se realizó el proceso de consentimiento informado personalmente en las instalaciones del hospital o en la casa del paciente y/o cuidador.

La intervención se realizó después de un día a tres días del alta hospitalaria, en la casa del cuidador y/o paciente. Se mostraron los videos en una tableta de siete pulgadas. Antes de comenzar con la intervención se mostró al cuidador un video que explicaba principalmente cuál era el lado afectado del paciente en los videos (video 0). El tiempo de visualización máximo para cada uno de los 8 videos fue de 30 minutos. Se vieron tres videos el primer día, los siguientes tres el segundo día y los dos últimos el tercer día. El investigador, quien era tecnóloga médico en terapia física y rehabilitación, estuvo presente durante toda la intervención para ayudar al cuidador en caso tuviera problemas con el manejo de la tableta y/o se presentara algún evento adverso.

Para las evaluaciones de las habilidades prácticas de los ocho videos, se pidió al cuidador que realizara cada actividad con el paciente tres veces. Cada ítem de la actividad se calificó como 1 si se realizó cómo se indica en al menos dos de las tres repeticiones, y 0 en caso contrario. Para la evaluación de los conocimientos se calificó como 1 cada respuesta correcta y 0 en caso contrario.

Primero, se evaluó las habilidades prácticas y los conocimientos para la actividad 1 (video 1). Segundo, los cuidadores vieron el video 1 ( $\sim 30 \mathrm{~min})$. Tercero, se volvió a evaluar las habilidades prácticas y los conocimientos para esa actividad. Esta secuencia se repitió para los videos 2 a 8 . Finalmente, después de los ocho videos, los cuidadores respondieron preguntas relacionadas a su satisfacción.

\section{Tamaño de muestra y análisis estadístico}

El cálculo del tamaño de muestra se realizó en base a un cambio cuantitativo en el puntaje de habilidades prácticas y conocimientos, por lo que el tamaño de muestra fue de solo 
10 participantes. Se consideró una diferencia media mínima para el nivel de habilidad práctica de 20 puntos y una desviación estándar máxima de 20 puntos ${ }^{(13)}$, la potencia $80 \%$ y el nivel de confianza $95 \%$.

Se verificó con la prueba de Shapiro-Wilk la distribución normal de las diferencias pos y pre para las habilidades prácticas y conocimientos, por lo que se utilizó la prueba $\mathrm{t}$ de Student para muestras pareadas para ambos casos. Se estableció un error alfa de $5 \%$ para todas las pruebas de hipótesis. Todos los análisis se realizaron en el programa estadístico Stata SE versión 15 (StataCorp LLC).

\section{Aspectos éticos}

Los Comité de Ética de la Universidad Peruana Cayetano Heredia (Constancia 124-04-18) y del Hospital Cayetano Heredia (Oficio No 1129-2018) aprobaron el estudio y el consentimiento informado. El cuidador y el paciente recibieron una copia del consentimiento informado firmado.

\section{RESULTADOS}

Desde agosto a diciembre de 2018, se contactó a los pacientes con ACV y a sus cuidadores. De 30 pacientes con ACV isquémico, 13 fueron excluidos, ocho porque presentaron condiciones cognitivas, neurológicas o psiquiátricas que no les permitían colaborar en las movilizaciones y transferencias; y cinco porque presentaron otros trastornos neurológi- cos, ortopédicos o traumatológicos que afectaban su movilidad. Los cuidadores de los 17 pacientes fueron elegibles, y siete se negaron a participar. Los 10 pacientes y sus cuidadores fueron asignados a los videos educativos, completaron la intervención y finalmente fueron analizados (Figura 1). Las características de los cuidadores y los pacientes se presentan en la Tabla 1. Hubo 10 parejas de cuidadores y pacientes. Todos los cuidadores eran mujeres y 9 de 10 de los pacientes eran hombres. La edad media de los pacientes fue mayor que la edad media de los cuidadores $(44,6 \pm 12,5$ frente a $67,9 \pm$ 11,4). 6 de 10 de los cuidadores cuidaban a sus padres y 6 de 10 de los pacientes tenían el lado derecho afectado.

Las habilidades prácticas y los conocimientos pre y posintervención se muestran en la Tabla 2. La diferencia pos-pre para las habilidades prácticas fue de 34,5 puntos ( $p$ $<0,001$ ), lo que representó un incremento del $50,7 \%$ en el puntaje, mientras que para los conocimientos fue de 10 puntos ( $p<0,001)$, es decir un incremento del 37\%.

En relación a la variable de satisfacción (Tabla 3). 7 de 10 de los cuidadores estaban muy satisfechos con los videos, 7 de 10 consideró fácil las palabras usadas y otros 7 consideró fácil poner en práctica las indicaciones. 9 cuidadores definitivamente recomendaría los videos y todos los consideraron útiles. Además, 2 de los cuidadores no tenía video favorito, mientras que otros prefirieron el video 2 - alineación en la posición echado de costado sobre el lado afectado, video 3 - alineación echado de costado sobre el lado sano y video 7 -

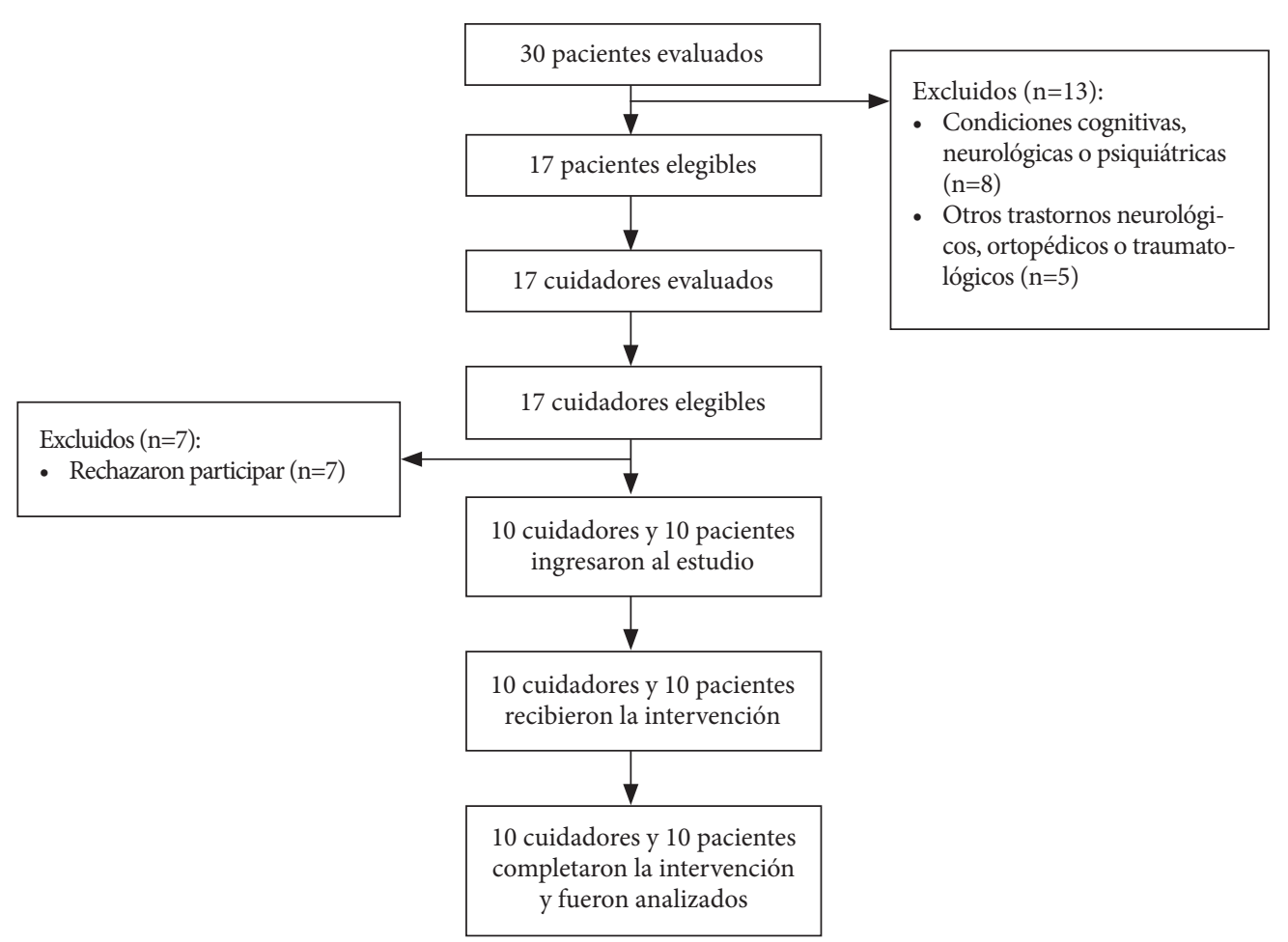

Figura 1. Flujograma de participantes del estudio. 
Tabla 1. Características de los cuidadores y pacientes en fase subaguda del accidente cerebrovascular.

\begin{tabular}{lcc}
\hline Características & $\begin{array}{c}\text { Cuidador } \\
\mathbf{n}=\mathbf{1 0}\end{array}$ & $\begin{array}{c}\text { Paciente } \\
\mathbf{n}=\mathbf{1 0}\end{array}$ \\
\hline Edad, media (DE) & $44,6(12,5)$ & $67,9(11,4)$ \\
Sexo & 0 & 9 \\
$\quad$ Masculino & 10 & 1 \\
$\quad$ Femenino & & \\
Grado de instrucción & 0 & 0 \\
$\quad$ Sin instrucción & 2 & 5 \\
$\quad$ Primaria & 2 & 4 \\
$\quad$ Secundaria & 4 & 0 \\
$\quad$ Superior no universitaria & 2 & 1 \\
$\quad$ Superior universitaria & & \\
Parentesco & 6 & \\
$\quad$ Hija & 2 & \\
$\quad$ Esposa & 1 & \\
$\quad$ Hermana & 1 & \\
$\quad$ Nuera & & \\
Lado afectado & & \\
$\quad$ Izquierdo & & \\
Derecho & &
\end{tabular}

DE: desviación estándar.

cómo trasladar al paciente de una cama a una silla. Algunos comentarios para el video 2 fueron: «descansa mejor el paciente» $\mathrm{y}$ «me parece perfecto para descansar»; para el video 3, «mi papá se siente más cómodo» $\mathrm{y}$ «es más cómodo»; para el video 7 , «fue más fácil hacerlo» y «es la posición más difícil de mover al paciente». Por otro lado, solo uno indicó que tenía un video no favorito (video 6 - cómo sentar al paciente en el borde de la cama desde la posición echada). Una observación para el video fue: «me pesa mucho».

Por otro lado, en el Material suplementario 4 se detalla el cambio en la habilidad práctica para cada ítem, mientras que en el Material suplementario 5 el cambio de conocimiento para cada pregunta. «No-No» significa que, en la pre y posevaluación, el ítem/respuesta fue incorrecto. «No-Sí» (lo que se espera encontrar con la intervención) indica que en la pre-evaluación el ítem/respuesta fue incorrecto, pero en la posevaluación fue correcto. «Sí-No» significa que en la pre-evaluación el ítem/respuesta fue correcto, pero en la pos- evaluación fue incorrecto. «Sí-Sí» indica que en la pre-evaluación y posevaluación el ítem/respuesta fue correcto.

\section{DISCUSIÓN}

Esta investigación muestra que los videos educativos mejoran las habilidades prácticas y los conocimientos de los cuidadores de pacientes en fase subaguda del ACV, específicamente sobre el posicionamiento, movilizaciones y transferencias. Por otro lado, los cuidadores tuvieron una percepción positiva de los videos y no se informaron eventos adversos durante la intervención.

El incremento en las habilidades prácticas fue similar a investigaciones que usaron videos educativos para mejorar tres técnicas de uso del inhalador en pacientes con EPOC $(22,7 \%, 22,9 \% \text { y } 12,8 \%)^{(13)}$, y a la capacitación presencial a los cuidadores de pacientes con ACV sobre medicación, ingesta de alimentos, posturas correctas, prevención de úlceras, entre otros $(16,7 \%)^{(20)}$. Aunque la mejora en las habilidades prácticas en este estudio fue mayor, esto podría deberse a que los pacientes con EPOC fueron en promedio 20 años mayores a la población de esta investigación $(67,7$ versus 44,6 años), lo que implicaría una curva de aprendizaje diferente. Además, debido a que la evaluación de la práctica en los cuidadores de pacientes con ACV capacitados presencialmente fue por medio del auto reporte de su práctica mientras que en este estudio a través de la evaluación del investigador con una lista de verificación.

En relación a los conocimientos, tanto la capacitación presencial de pacientes de ACV ${ }^{(20)}$ como los videos en cuidadores de pacientes con asma ${ }^{(15)}$, niños con epilepsia ${ }^{(16)} \mathrm{y}$ pacientes en cuidados intensivos ${ }^{(17)}$, han incrementado los conocimientos sobre la patología, condición de salud, o manejo, a pesar que para la evaluación de los conocimientos se haya usado adaptaciones de cuestionarios validados, cuestionarios validados, o cuestionarios desarrollados para la investigación sin un proceso de validación formal. En algunos estudios no se han considerado el nivel de instrucción de los cuidadores, y en otros, al igual que en éste, se ha encontrado que la mayoría de cuidadores tenían al menos el nivel secun-

Tabla 2. Habilidades prácticas y conocimientos de los cuidadores sobre posicionamientos, movilizaciones y transferencias para pacientes en fase subaguda del accidente cerebrovascular.

\begin{tabular}{lccccc}
\hline \multirow{2}{*}{ Medición en el cuidador } & \multicolumn{2}{c}{ Media (DE) } & \multicolumn{2}{c}{$\begin{array}{c}\text { Diferencia entre las medias } \\
(\text { IC 95\%) }\end{array}$} & Valor de p \\
\cline { 2 - 3 } & Pre & Pos & & $34,5(29,0-40,1)$ & $<0,001^{*}$ \\
Nivel de práctica & $21,6(5,5)$ & $56,1(6,9)$ & & $10,0(7,4-12,6)$ & $<0,001^{*}$ \\
Nivel de conocimiento & $11,6(3,7)$ & $21,6(3,9)$ & & $10,9)$ \\
\hline
\end{tabular}

DE: desviación estándar; IC: intervalo de confianza.

${ }^{*}$ t de Student para muestras pareadas. 
dario. Por lo que, este tipo de intervenciones podría tener diferentes efectos en los conocimientos de los participantes de acuerdo a su nivel de instrucción.

Es importante evaluar la satisfacción de los cuidadores para conocer su experiencia en relación a los videos. Se encontraron resultados positivos en otras investigaciones de un solo grupo con pretest y postest ${ }^{(14,23)}$; sin embargo, en estudios cuasi-experimentales con grupo control no equivalente, los videos no han demostrado superioridad sobre otros métodos, por ejemplo, el agregar videos a un enfoque educativo integral no cambia el nivel de satisfacción ${ }^{(24)}$, ni los cuidadores notan diferencias entre la utilidad de los videos o instrucciones en papel ${ }^{(25)}$.

Esta investigación no evaluó indicadores clínicos a diferencia de algunos estudios que han determinado que los videos antes de un procedimiento de quimioterapia intratecal disminuyen los niveles de ansiedad ${ }^{(26)}$ o el número de complicaciones en pacientes con secuela de ACV (27). Sin embargo, según el modelo de Kirkpatrick, que evalúa los programas de entrenamiento en 4 niveles; la satisfacción, conocimientos y las habilidades prácticas corresponden a los dos primeros niveles (nivel de reacción y nivel de aprendizaje), que son necesarios e importantes para impactar en niveles superiores (nivel de comportamiento y nivel de resultados) ${ }^{(28)}$. Por otro lado, se tomó en cuenta teorías y principios para el desarrollo de los videos a fin de maximizar el aprendizaje del cuidador, entre ellas, teorías como la carga cognitiva y la teoría cognitiva del aprendizaje multimedia; y principios como señalización, segmentación, deshierbe y modalidad de emparejamiento ${ }^{(29)}$.

Este estudio presenta algunas limitaciones, por lo que los resultados deben interpretarse con cautela. Primero, el sesgo de observación, debido a que el investigador fue el que realizó la evaluación pre y pos, lo que podría explicar que la diferencia en las habilidades prácticas fue mayor al de los conocimientos. Segundo, la presencia del investigador podría tener una influencia positiva en la habilidad práctica de los cuidadores porque se sentían observados o más seguros. Sin embargo, las diferencias encontradas en las habilidades prácticas y conocimientos fueron altas; $50,7 \%$ y $37 \%$, respectivamente. Tercero, no hay un grupo de control para identificar el efecto real de la intervención; sin embargo, es poco probable que la dramática mejora observada tanto en práctica como en conocimiento en un breve tiempo pueda explicarse por tendencias seculares, o por la adquisición gradual de experiencia en el manejo de estos pacientes. Cuarto, el tamaño de muestra fue pequeño, puesto que la estimación se realizó en base a una diferencia esperada de 20 puntos; sin embargo, no hay evidencia que sea un valor aceptado como relevante para ver cambios. Quinto, los instrumentos usados fueron de elaboración propia y no tuvieron validación formal, aunque se desarrollaron en base a literatura especializada, juicio de expertos y un piloto. En sexto lugar, no hubo seguimiento para evaluar el efecto a lo largo del tiempo, aun-
Tabla 3. Satisfacción de los cuidadores en relación a los videos.

\begin{tabular}{|c|c|}
\hline Dimensión & $\mathbf{n}$ \\
\hline \multicolumn{2}{|l|}{ Satisfacción } \\
\hline Satisfecho & 3 \\
\hline Muy satisfecho & 7 \\
\hline \multicolumn{2}{|l|}{ Palabras usadas en los videos } \\
\hline Muy difíciles & 1 \\
\hline Fáciles & 7 \\
\hline Muy fáciles & 2 \\
\hline \multicolumn{2}{|l|}{ Práctica de lo observado } \\
\hline Ni fácil ni difícil & 3 \\
\hline Fácil & 7 \\
\hline \multicolumn{2}{|l|}{ Recomendaría los videos } \\
\hline Probablemente sí & 1 \\
\hline Definitivamente sí & 9 \\
\hline \multicolumn{2}{|l|}{ Utilidad de los videos } \\
\hline Definitivamente sí & 10 \\
\hline \multicolumn{2}{|l|}{ Video preferido } \\
\hline No tengo preferencias & 2 \\
\hline Video 2 & 2 \\
\hline Video 3 & 2 \\
\hline Video 5 & 1 \\
\hline Video 6 & 1 \\
\hline Video 7 & 2 \\
\hline \multicolumn{2}{|l|}{ Video menos preferido } \\
\hline No tengo preferencias & 9 \\
\hline Video 6 & 1 \\
\hline
\end{tabular}

que es probable que el conocimiento tienda a disminuir ${ }^{(30)}$ e incluso las habilidades prácticas. Séptimo, la evaluación se realizó inmediatamente después de la intervención, lo que no necesariamente significa que los cuidadores pongan en práctica lo que aprendieron; sin embargo, según el modelo de Kirkpatrick, es necesaria la evaluación realizada para evaluar posteriormente el cambio de comportamiento.

Los videos educativos que se han desarrollado podrían usarse para capacitar a los cuidadores sobre el manejo de pacientes con ACV, e incluso ser parte de un programa de capacitación integral continua que también incluya capacitación presencial, práctica en pacientes simulados, entre otras estrategias. Por otro lado, si bien los videos han sido desarrollados para pacientes con ACV con ciertas características clínicas, estos videos podrían ser útiles en otras condiciones de ACV, según indicación de los especialistas; e incluso en otros tipos de pacientes cuya movilidad esté afectada, por ejemplo, en pacientes geriátricos, pacientes en cuidados paliativos y hospitalizados. Las ventajas de los videos es que 
son herramientas prácticas, no caras una vez producidas, las personas pueden aprender a su propio ritmo, y muchas personas pueden acceder a los videos al mismo tiempo sin que necesariamente esté presente un profesional de la salud.

Los videos educativos mejoran las habilidades prácticas y los conocimientos de los cuidadores informales de pacientes con accidente cerebrovascular en fase subaguda. Además, los cuidadores recibieron positivamente los videos, estaban muy satisfechos, consideraron fáciles las palabras de los videos y poner en práctica las indicaciones, definitivamente sí los recomendarían y los consideran útiles. Se recomienda desarrollar versiones mejoradas de los videos en base al análisis que se ha realizado por cada uno de los ítems y preguntas para las habilidades prácticas y conocimientos, respectivamente. Asimismo, realizar estudios que hagan seguimiento a largo plazo de los indicadores evaluados y que evalúen el impacto de la capacitación, por ejemplo, en indicadores clínicos en los pacientes y en indicadores de salud mental en los cuidadores.

\section{REFERENCIAS BIBLIOGRÁFICAS}

1. Hewer RL. Motor loss and swallowing difficulty after stroke: frequency, recovery, and prognosis. Acta Neurol Scand. 1987;76(1):50-4. doi:10.1111/j.1600-0404.1987.tb03543.x.

2. Zawawi NSM, Aziz NA, Fisher R, Ahmad K, Walker MF. The Unmet Needs of Stroke Survivors and Stroke Caregivers: A Systematic Narrative Review. J Stroke Cerebrovasc Dis. 2020;29(8):104875. doi:10.1016/j. jstrokecerebrovasdis.2020.104875.

3. Málaga G, De La Cruz-Saldaña T, Busta-Flores P, Carbajal A, Santiago-Mariaca K. La enfermedad cerebrovascular en el Perú: estado actual y perspectivas de investigación clínica. Acta Med Peru. 2018;35(1):51-4.

4. Levin PG. Stronger after stroke: your roadmap to recovery [Internet]. $3^{\circ}$ ed. New York: Springer; 2018 [citado el 5 de noviembre de 2018]. Disponible en: https://books.google.com.pe/books?id=Vt1JDwAAQBAJ\&pg=PA146\&dq=stroke + phases + acute + subacute + chroni$c \& h l=e s \& s a=X \& v e d=0$ ahUKEwj-8fyS6L3eAhUm0FkKHVKN D8UQ6AEIJzAA\# $\mathrm{v}=$ onepage \&q=stroke phases acute subacute chronic\&f=false

5. Brereton L, Carroll C, Barnston S. Interventions for adult family carers of people who have had a stroke: A systematic review. Clin Rehabil. 2007;21(10):867-84. doi:10.1177/0269215507078313.

6. Pitthayapong S, Thiangtam W, Powwattana A, Leelacharas S, Waters CM. A Community Based Program for Family Caregivers for Post Stroke Survivors in Thailand. Asian Nurs Res (Korean Soc Nurs Sci). 2017;11(2):150-7. doi:10.1016/j.anr.2017.05.009.

7. Pesantes MA, Brandt LR, Ipince A, Miranda JJ, Diez-Canseco F. An exploration into caring for a stroke-survivor in Lima, Peru: Emotional impact, stress factors, coping mechanisms and unmet needs of informal caregivers. eNeurologicalSci. 2017;6:33-50. doi:10.1016/j. ensci.2016.11.004.

8. Chatterton HJ, Pomeroy VM, Gratton J. Positioning for stroke patients: A survey of physiotherapists' aims and practices. Disabil Rehabil. 2001;23(10):413-21. doi:10.1080/09638280010008825.

9. Gillen G. Stroke Rehabilitation: A Function-based Approach [Internet]. $4^{\circ}$ ed. United States of America: Elsevier; 2015 [citado el 22 de enero de 2021]. Disponible en: https://books.google.com.pe/books?id=Id4vCgAAQBAJ\&printsec $=$ frontcover\&dq=Stroke+Rehabilitation:+A+-
Contribuciones de los autores: CSH participó de la concepción y diseño del artículo; recolección de resultados; análisis e interpretación de datos; redacción del artículo y revisión crítica del artículo. CCC participó de la concepción y diseño del artículo; análisis e interpretación de datos; revisión crítica del artículo; y asesoría estadística. Ambos autores aprobaron la versión final y asumen la responsabilidad frente a todos los aspectos del manuscrito.

Financiamiento: Este trabajo fue financiado por CONCYTEC (Consejo Nacional de Ciencia, Tecnología e Innovación Tecnológica), Lima-Perú. Esta institución proporcionó una beca a Claudia María Sánchez-Huamash para estudiar una maestría, y apoyo financiero para la realización de la investigación. Sin embargo, no participó en el diseño del estudio, la recopilación, el análisis y la interpretación de los datos, la redacción del informe, ni la decisión de enviar el artículo para su publicación.

Conflictos de interés: Los autores no tienen ningún conflicto de interés que declarar.

Material suplementario: Disponible en la versión electrónica de la RPMESP.

Function-based + Approach\&hl=es-419\&sa $=X \& v e d=0$ ahUKEwiY6by83cLmAhVRA9QKHSvPDDoQ6wEILzAA\#v=onepage\&q=Stroke Rehabilitation\%3A A Function-based Approach\&f=false

10. Avezum Á, Costa-Filho FF, Pieri A, Martins SO, Marin-Neto JA. Stroke in Latin America: Burden of Disease and Opportunities for Prevention. Glob Heart. 2015;10(4):323-31. doi:10.1016/j.gheart.2014.01.006.

11. Lazo-Gonzales O, Alcalde-Rabanal J, Espinosa-Henao O. El sistema de salud en Perú: situación y desafíos [Internet]. Lima: Colegio Médico del Perú, REP; 2016 [citado el 18 de enero de 2021]. Disponible en: http://web2016. cmp.org.pe/wp-content/uploads/2016/12/libroSistemaSaludPeru-.pdf

12. Miranda JJ, Moscoso MG, Yan LL, Diez-Canseco F, Málaga G, Garcia $\mathrm{HH}$, et al. Addressing post-stroke care in rural areas with Peru as a case study. Placing emphasis on evidence-based pragmatism. J Neurol Sci. 2017;375:309-15. doi:10.1016/j.jns.2017.02.027.

13. Mulhall AM, Zafar MA, Record S, Channell H, Panos RJ. A Tablet-Based Multimedia Education Tool Improves Provider and Subject Knowledge of Inhaler Use Techniques. Respir Care. 2017;62(2):16371. doi:10.4187/respcare.05008.

14. Denny MC, Vahidy F, Vu KYT, Sharrief AZ, Savitz SI. Video-based educational intervention associated with improved stroke literacy, selfefficacy, and patient satisfaction. PLoS One. 2017;12(3):e0171952. doi:10.1371/journal.pone.0171952.

15. Riera A, Ocasio A, Tiyyagura G, Thomas A, Goncalves P, Krumeich $\mathrm{L}$, et al. A web-based educational video to improve asthma knowledge for limited English proficiency Latino caregivers. J Asthma. 2017;54(6):624-31. doi:10.1080/02770903.2016.1251597.

16. Saengow VE, Chancharoenchai P, Saartying W, Pimpa W, Chotichanon $\mathrm{N}$, Lewsirirat $\mathrm{T}$, et al. Epilepsy video animation: Impact on knowledge and drug adherence in pediatric epilepsy patients and caregivers. Clin Neurol Neurosurg. 2018;172:59-61. doi:10.1016/j. clineuro.2018.06.031.

17. Krewulak KD, Bull MJ, Wesley Ely E, Davidson JE, Stelfox HT, Fiest KM. Effectiveness of an intensive care unit family education intervention on delirium knowledge: a pre-test post-test quasi-experimental study. Can J Anesth. 2020;67(12):1761-74. doi:10.1007/s12630-02001810-5. 
18. Tan ZS, Soh M, Knott A, Ramirez K, Ercoli L, Caceres N, et al. Impact of an Intensive Dementia Caregiver Training Model on Knowledge and Self-Competence: The Improving Caregiving for Dementia Program. J Am Geriatr Soc. 2019;67(6):1306-9. doi:10.1111/jgs.15821.

19. Hendrix CC, Bailey DE, Steinhauser KE, Olsen MK, Stechuchak KM, Lowman SG, et al. Effects of enhanced caregiver training program on cancer caregiver's self-efficacy, preparedness, and psychological well-being. Support Care Cancer. 2016;24(1):327-36. doi:10.1007/ s00520-015-2797-3.

20. Choi JS, Seo YM, Kwon IS. Effects of education on knowledge and practice of caregivers of the stroke patient. J Korean Acad Nurs. 2006;36(7):1175-1182. doi:10.4040/jkan.2006.36.7.1175.

21. Winstein CJ, Stein J, Arena R, Bates B, Cherney LR, Cramer SC, et al. Guidelines for Adult Stroke Rehabilitation and Recovery: A Guideline for Healthcare Professionals from the American Heart Association/American Stroke Association. Stroke. 2016;47(6):e98-169. doi:10.1161/STR.0000000000000098.

22. Davies PM, Torres Lacomba M. Pasos a seguir: tratamiento integrado de pacientes con hemiplejía. $2^{\circ}$ ed. Madrid: Médica Panamericana; 2008.

23. Vogler CN, Sattovia S, Salazar LY, Leung TI, Botchway A. Assessing outcomes of educational videos in group visits for patients with chronic pain at an academic primary care clinic. Postgrad Med. 2017;129(5):524-30. doi:10.1080/00325481.2017.1324228.
24. Meighan MM. Stroke Education Video Does Not Affect Patient Satisfaction Scores: A System Analysis. J Neurosci Nurs. 2018;50(4):233-7. doi:10.1097/JNN.0000000000000383.

25. Wood EB, Harrison G, Trickey A, Friesen MA, Stinson S, Rovelli E, et al. Evidence-Based Practice: Video-Discharge Instructions in the PediatricEmergency Department. JEmerg Nurs. 2017;43(4):316-21. doi:10.1016/j.jen.2016.11.003.

26. Bany Hamdan A, Ballourah W, Elghazaly A, Javison S, Alshammary S, Erlandez R, et al. The Effect of Video-Assisted Education Prior Intrathecal Chemotherapy on Anxiety and Knowledge Enhancement. J Cancer Educ. 2020;28(1):e12113. doi:10.1007/s13187-020-01787-1.

27. Kamal A, Khoja A, Usmani B, Magsi S, Malani A, Peera Z, et al. Effect of 5-minute movies shown via a mobile phone app on risk factors and mortality after stroke in a low- To middle-income country: Randomized controlled trial for the stroke caregiver dyad education intervention (MovIes4Stroke). JMIR mHealth uHealth. 2020;8(1). doi:10.2196/12113.

28. Smidt A, Balandin S, Sigafoos J, Reed VA. The Kirkpatrick model: A useful tool for evaluating training outcomes. J Intellect Dev Disabil. 2009;34(3):266-74. doi:10.1080/13668250903093125.

29. Brame CJ. Effective educational videos: Principles and guidelines for maximizing student learning from video content. CBE Life Sci Educ. 2016;15(4):es6. doi:10.1187/cbe.16-03-0125.

30. Chan YF, Lavery R, Fox N, Kwon R, Zinzuwadia S, Massone R, et al. Effect of an Educational Video on Emergency Department Patient Stroke Knowledge. J Emerg Med. 2008;34(2):215-20. doi:10.1016/j. jemermed.2007.04.003. 